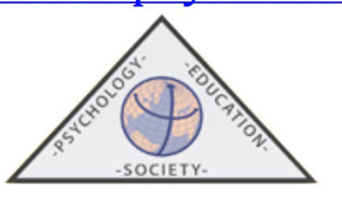

\title{
Las funciones ejecutivas como predictoras del rendimiento académico de estudiantes universitarios
}

\author{
Karol GUTIÉRREZ-RUIZ, Joel PATERNINA, Sharick ZAKZUK, Sandra MENDEZ, \\ Angie CASTILLO, Laura PAYARES y Andrea PEÑATE
}

\author{
Universidad Tecnológica de Bolívar
}

(Recibido el 9 de Agosto de 2018, Aceptado el 29 de Julio de 2020)

RESUMEN: En este trabajo se estudia la relación entre Funciones Ejecutivas (FE) y rendimiento académico, analizando mediante medidas cognitivas de FE si la memoria de trabajo, el control inhibitorio, la flexibilidad cognitiva, y la capacidad de abstracción y formación de conceptos predicen el logro académico general de estudiantes universitarios. Se realizó un estudio no experimental transversal correlacional-causal en el que participaron 104 estudiantes universitarios, quienes fueron evaluados mediante el Test de clasificación de tarjetas de Wisconsisn modificado (M-WCST), el Test de Colores y Palabras Stroop, y el índice de Memoria de trabajo de la Escala de inteligencia para adultos de Wechsler WAIS-III; y se registró su promedio acumulado de calificaciones del último periodo académico cursado. Se realizó un análisis de regresión jerárquica múltiple para evaluar si las FE contribuyen significativamente al logro académico general, y se comparó el desempeño en tareas de FE entre estudiantes con rendimiento académico bajo y promedio. Los resultados mostraron que la memoria de trabajo verbal, la flexibilidad cognitiva y la capacidad de abstracción y formación de conceptos predicen el rendimiento académico general de los estudiantes universitarios, aunque logran explicar solo el 19,8\% de la varianza. Se encontró diferencia estadísticamente significativa con un tamaño del efecto mediano entre el grupo con rendimiento académico bajo y promedio en la tarea de memoria de trabajo verbal Aritmética. Se plantea la necesidad de considerar la edad como posible variable mediadora de la relación entre FE y rendimiento académico general, y de explorar otras variables cognitivas, conductuales y psicosociales que permitan predecir en mayor medida el rendimiento académico de estudiantes universitarios.

Palabras claves: funciones ejecutivas; rendimiento académico; control inhibitorio; memoria de trabajo; flexibilidad cognitiva.

\section{The Executive Functions As Predictors of the Academic Performance of College Students}

\begin{abstract}
In this paper, we study the relationship between Executive Functions (EF) and academic performance, analyzing by means of EF cognitive measures if working memory, inhibitory control, cognitive flexibility and categorization predict the general academic achievement of college students. A non-experimental cross-sectional study was conducted involving 104 college, who were evaluated using the Modified Wisconsisn Card Sorting Test (M-WCST), the Stroop color and word test, and working
\end{abstract}


memory index from Wechsler Adult Intelligence Scale WAIS-III; and the cumulative grade point average for the last academic period was recorded. A multiple hierarchical regression analysis was conducted to evaluate whether EFs contribute significantly to overall academic achievement, and the performance of EF tasks among students with low and average academic performance was compared. The results showed that the verbal working memory, cognitive flexibility and the abstraction and concept formation predict the general academic performance of college students, although they manage to explain 19,8\% of the variance. A statistically significant difference was found with a median effect size between the group with low and average academic performance in the verbal working memory task. It is proposed the need to consider age as a possible mediating variable of the relationship between EF and general academic performance, and to explore other cognitive, behavioral and psychosocial variables that could be influencing in the academic performance of college students.

Keywords: Executive Functions; Academic Performance; Inhibitory Control; Working Memory; Cognitive Flexibility.

Correspondencia: Karol Gutiérrez-Ruiz. Parque Industrial y Tecnológico Carlos Vélez Pombo Km 1 Vía Turbaco, Bolívar, Colombia. Email: kgutierrez@utb.edu.co

\section{Introducción}

En el ámbito educativo ha crecido el interés por estudiar los determinantes del rendimiento académico incluyendo variables cognitivas implicadas en los procesos de aprendizaje que pudieran explicar el fracaso escolar (Cerda, Pérez, Romera, Casas, \& Ortega-Ruiz, 2017; Iniesta, López-López, Corbí, Pérez, \& Costa, 2017). Estas variables cognitivas están relacionadas con procesos de maduración cerebral que ocurren a lo largo del ciclo vital (Gómez-Pérez, Ostrosky-Solís, \& Próspero-García, 2003).

Estudios en adolescentes evidencian que el desarrollo del cerebro puede continuar hasta alrededor de los 25 años de edad, y que cambios importantes en el funcionamiento neuropsicológico, en las emociones, procesos de cognición social y en el comportamiento parecen estar asociados a la maduración de estructuras y redes cerebrales (Casey, Getz, \& Galvan, 2008). Por ejemplo, el córtex prefrontal y sus conexiones se encuentran aún en proceso de maduración durante la adolescencia y la adultez temprana (Giedd \& Rapoport, 2010), lo que implica que el rendimiento académico general en la educación superior podría estar influenciado por la etapa de maduración cerebral y desarrollo cognitivo de los estudiantes (Baars, Nije Bijvank, Tonnaer, \& Jolles, 2015).

Se presume que las Funciones Ejecutivas (FE) son variables cognitivas que juegan un papel importante en el desarrollo y mantenimiento de habilidades académicas, y en el consecuente rendimiento académico general. Esto ha sido principalmente estudiado en la educación primaria y secundaria, sin embargo, son pocas las investigaciones encaminadas a determinar el impacto del funcionamiento ejecutivo en el desempeño de estudiantes en el contexto universitario. De acuerdo con Santa-Cruz y Rosas (2017), las FE incluyen aquellas habilidades que nos permiten trazar metas y alcanzarlas a través de planes de acción, y el monitoreo de la evolución de los mismos, ignorando o resistiendo pensamientos, conductas y emociones que interfieren en el logro de los objetivos propuestos. 
El modelo de Miyake et al. (2000), el cual ha recibido amplio respaldo empírico, propone que son tres los dominios de las FE: Control inhibitorio, memoria de trabajo y flexibilidad cognitiva; los cuales están interrelacionados. El control inhibitorio se considera como un mecanismo para detectar o suprimir respuestas cognitivas o conductuales predominantes y en ocasiones automáticas cuando la situación lo requiere (García-Molina, Enseñat, Tirapu-Ustárroz, \& Roig-Rovira, 2009). Una tarea de inhibición prototípica es la tarea de Stroop, en la que se necesita inhibir o anular la tendencia a producir una respuesta más dominante o automática.

La memoria de trabajo se define como un sistema multimodal que permite mantener información en la memoria durante un periodo de tiempo y manipularla mentalmente con la ayuda de procesos atencionales para resolver un problema (Baddeley, 2012). Esta capacidad es muy importante porque está asociada con el aprendizaje, el seguimiento de instrucciones, y con el sostenimiento de la atención; y parece no estar afectada por las experiencias educativas previas, el estatus socioeconómico o la pertenencia a un grupo étnico (Gathercole, \& Alloway, 2008; Prosen \& Smrtnik Vitulić, 2014).

Por su parte, la flexibilidad cognitiva "implica la habilidad para cambiar entre un set de respuestas diferentes ya sea de pensamientos o de acciones en dependencia de las demandas de la situación" (Monsell, 2003, citado por Ramírez, \& Ostrosky, 2012, p.583). La flexibilidad cognitiva es un proceso muy importante para el aprendizaje y regulación del pensamiento y la acción, su alteración dificulta aprender de los errores y generar estrategias alternativas, incluso dividir la atención y procesar la información actual (Anderson, 2002, citado por Ramírez, \& Ostrosky, 2012, p. 583).

Se afirma que las FE son ingredientes esenciales para el funcionamiento académico óptimo y una de las principales causas de las dificultades escolares en la infancia (Rose \& Rose, 2007). En relación a esto, Visu-Petra, Cheie, Benga, y Miclea (2011) encontraron relación significativa entre el rendimiento académico de niños entre quinto y octavo grado de una escuela del noreste de Rumania, y su desempeño en tareas de memoria de trabajo visoespacial e inhibición. Reyes, Barreyro, e Injoque-Ricle (2015) estudiaron el rol de las FE en el rendimiento académico en 101 niños de 9 años de edad, encontrando que éstas predicen significativamente el rendimiento académico global y que las más correlacionadas con éste son la memoria de trabajo, la fluidez verbal, la atención sostenida, la atención selectiva y la planificación.

En el estudio realizado por Samuels, Tournaki, Blackman, \& Zilinski (2016) se afirma que las FE de estudiantes de educación media (sexto a noveno grado, con edades entre 12 y 15 años) pueden ser predictores a largo plazo del rendimiento académico en la secundaria. En este trabajo se correlacionó el promedio de calificaciones de los cursos de inglés, artes, matemáticas, estudios sociales, ciencia y español; y los resultados obtenidos en el instrumento Evaluación conductual de las Funciones Ejecutivas (BRIEF), una medida de autorreporte. Por su parte, Ramos, Jadán-Guerrero, y Gómez-García (2018) utilizaron el instrumento de autorreporte Escala para evaluar Funciones Ejecutivas (EFECO) en niños de educación secundaria entre 12 y 18 años encontrando que la flexibilidad cognitiva, organización de materiales, monitorización, iniciativa, memoria de trabajo, y planificación presentan una relación significativa e inversamente proporcional con el rendimiento académico. Adicionalmente, Simone, Marks, Bédard, y Halperin (2018) reportan que la memoria de trabajo auditiva-verbal y visoespacial influyen de manera 
significativa en el rendimiento escolar general de niños de 8 años, principalmente debido a su relación con el desarrollo de habilidades académicas como lecto-escritura y aritmética.

En el contexto universitario, Knouse, Feldman y Blevins (2014) aplicaron la Escala Barkley de déficit en las Funciones Ejecutivas (Barkley, 2012), una medida de autorreporte, concluyendo que las FE son útiles para predecir el rendimiento académico futuro de los estudiantes, principalmente el componente de auto-motivación. Por su parte, Baars et al., (2015) estudiaron la asociación entre rendimiento académico y tres medidas conductuales de FE en 1332 estudiantes de primer año de educación superior. Utilizaron cuestionarios de autorreporte de atención, planificación, y autocontrol/automonitorización. Los resultados mostraron que los estudiantes con mejor autoevaluación de FE obtuvieron más créditos de estudio al final del año académico que los estudiantes con una autoevaluación de FE inferior. Los autores afirman que se espera que estudiantes con niveles más bajos de regulación atencional, planificación, y autocontrol/ automonitorización, tengan mayores problemas en la planificación del estudio y en el monitoreo del progreso del estudio, lo que afectaría su desempeño académico. Finalmente, McKee (2017) estudió la relación entre rendimiento académico y FE mediante el Test de Clasificación de Tarjetas de Wisconsin (WCST) y el cuestionario de autorreporte de FE Webexec, encontrando que el desempeño en estas medidas predice el rendimiento académico en estudiantes universitarios. Este es, hasta donde sabemos, uno de los pocos trabajos realizados en población universitaria en el que se incluye una medida cognitiva de las FE, el WCST.

Teniendo en cuenta lo anterior, programas de bienestar universitario han mostrado interés en las FE como posibles predictoras del logro académico general, asumiendo que intervenir en estas habilidades cognitivas en el contexto universitario podría tener un impacto positivo en el desempeño académico de los estudiantes y a su vez en los indicadores de permanencia. Sin embargo, los resultados de algunos estudios sobre la relación entre FE y rendimiento académico han sido contradictorios, ejemplo de esto son los trabajos de Barceló, Lewis y Moreno (2006), Dubuc, Aubertin-Leheudre, y Karelis (2020), Espy et al. (2004), y Van der Ven, Kroesbergen, Boom y Leseman (2012); lo que indica que éste debe seguir siendo un tema objeto de investigación.

En el trabajo realizado por Barceló et al. (2006) se concluye que el rendimiento académico de estudiantes universitarios no está directamente relacionado con déficits en FE. En este estudio se incluyen como componentes de las FE el control inhibitorio, la fluidez verbal y la flexibilidad cognitiva; y se utilizan medidas cognitivas de estos procesos. Espy et al. (2004) encontraron que el rendimiento académico guarda una fuerte asociación con el control inhibitorio, y una baja asociación con la memoria de trabajo y la flexibilidad cognitiva. En tanto que en el estudio de Van der Ven et al. (2012), se encontró que el rendimiento académico está relacionado con la memoria de trabajo, pero no con el control inhibitorio y la flexibilidad cognitiva. Por su parte, Dubuc et al. (2020) analizaron la relación entre el control de interferencia y la memoria de trabajo con el rendimiento académico, encontrando que estas variables tienen una debil asociación entre sí y por tanto tienen una limitada capacidad para predecir el rendimiento académico.

Considerando lo anterior, el presente trabajo tiene como objetivo estudiar la relación entre FE y rendimiento académico, analizando mediante medidas cognitivas de FE si la memoria de trabajo, el control inhibitorio, la flexibilidad cognitiva y la categorización predicen el logro académico general de estudiantes universitarios. Para esto se caracteriza inicialmente el funcionamiento ejecutivo de estudiantes universitarios, se establece la asociación entre las medidas 
cognitivas de FE y el rendimiento académico, determinando su poder predictivo mediante un análisis de regresión linear; y se analizan las diferencias entre grupos conformados en función del sexo, y entre grupos con rendimiento académico promedio y bajo rendimiento académico en las medidas cognitivas de FE.

\section{Método}

\section{Participantes}

Un total de 104 sujetos participaron en el estudio, 72 varones $\left(\mathrm{M}_{\text {edad }}=19.6\right.$. DT=1.9) y 32 mujeres $\left(\mathrm{M}_{\text {edad }}=18.8\right.$. DT=1.2), estudiantes de las facultades de Ciencias sociales y humanidades, Ingeniería, y Economía y negocios de la Universidad Tecnológica de Bolívar en Cartagena, Colombia. Los sujetos fueron seleccionados mediante un muestreo no probabilístico. Participaron de forma anónima y voluntaria, sin retribución alguna, y podían retirarse del estudio en cualquier momento. Todos los participantes eran mayores de edad, su lengua nativa era el español, carecían de deficiencias visuales y/o auditivas no controladas, y no tenían antecedentes de patología neurológica o psiquiátrica.

\section{Instrumentos}

Control inhibitorio. Test de colores y palabras Stroop (Golden, 1999). El instrumento es una medida de la capacidad inhibitoria y de la resistencia a la interferencia. Está conformado por tres tareas: (a) lectura de palabra, (b) denominación de colores y (c) tarea de interferencia. En ésta última el participante debe inhibir la lectura de la palabra para dar lugar a la denominación del color. Las puntuaciones normalizadas en esta tarea se expresan como puntuaciones típicas, con una media de 50 y una desviación estandar de 10. La fiabilidad de este instrumento es adecuada, con un coeficiente de confiabilidad superior a 0.70 en todas las láminas (Barreto, Roa, \& del Carmen Pulido, 2016).

Memoria de Trabajo. Se calculó el índice de memoria de trabajo (IMT) de la Escala de Inteligencia para adultos de Wechsler-III (WAIS-III; Wechsler, 2001). Este índice incluye tres subpruebas: dígitos en orden directo e inverso, letras números y aritmética. Se trata de una medida de memoria de trabajo verbal. Las puntuaciones normalizadas en esta tarea se expresan para el IMT como CI, con una media de 100 y una desviación estandar de 15. En el caso de las subpruebas que conforman el índice, se trata de puntuaciones escalares con una media de $10 \mathrm{y}$ una desviación estandar de 3. La fiabilidad de este instrumento es adecuada, con un coeficiente de confiabilidad igual a 0.92 .

Flexibilidad cognitiva y Categorización. Test de clasificación de tarjetas de Wisconsin modificado (M-WCST; Heaton, Chelune, Talley, Kay, \& Curtiss. 1993). La prueba permite calcular el número de categorías, perseveraciones y errores totales. Las puntuaciones normalizadas en esta tarea se expresan como puntuaciones típicas, con una media de 50 y una desviación estandar de 10. El instrumento cuenta con datos normativos para población colombiana de niños/as entre los 6 y 17 años de edad (Arango-Lasprilla, et al., 2017). La fiabilidad de este instrumento es adecuada, con un coeficiente de confiabilidad igual a 0.83 . 


\section{Procedimiento}

El presente estudio contó con el aval del Comité de investigaciones de la Universidad Tecnológica de Bolívar. Todos los sujetos participaron voluntariamente y firmaron un consentimiento informado, y se les informó que podían desistir en cualquier momento de su participación. De igual manera, se comunicó que los datos serían codificados mediante números para evitar la identificación de los participantes y de su carácter confidencial.

Los participantes fueron evaluados mediante la administración de los instrumentos de manera individual en el área de Neurociencias del Laboratorio de Psicología y se registró el promedio académico acumulado del semestre inmediatamente anterior.

\section{Análisis de datos}

Se realizó un análisis de regresión jerárquica múltiple utilizando como variable dependiente el promedio académico acumulado del semestre inmediatamente anterior e introduciendo las variables cognitivas de FE en sucesivos pasos; esto con el fin de evaluar si las FE predicen significativamente el logro académico general. Teniendo en cuenta que se ha demostrado en diferentes estudios que la memoria de trabajo influye en el rendimiento escolar (Visu-Petra et al., 2011; Samuels et al., 2016), se introdujo como primera variable en el modelo para controlar su efecto sobre la variable dependiente.

Adicionalmente, se analizaron diferencias entre grupos en función del sexo de los participantes y de su rendimiento académico (promedio y bajo rendimiento). Las comparaciones de grupos se realizaron mediante el estadístico U de Mann Whithney, estimando el tamaño del efecto para las diferencias estadísticamente significativas. Se empleó una prueba no paramétrica como la U de Mann Whithney debido a que los datos no se distribuian normalmente, el contraste de normalidad se realizó mediante la prueba Kolmogorov-Smirnov $(\mathrm{p}<.05)$. Para establecer los grupos en función del rendimiento académico se tuvo en cuenta que las calificaciones en el Sistema de Educación Superior colombiano están expresadas en una escala numérica creciente de 0.0 a 5.0. La puntuación mínima para superar la materia es 3.5 y la puntuación máxima es 5.0, así, en este trabajo los estudiantes con promedio menor a 3.5 fueron clasificados como estudiantes con bajo rendimiento académico. Los análisis de datos se realizaron con el paquete estadístico para las ciencias sociales (SPSS) versión 24.0 para Windows.

\section{Resultados}

En la Tabla 1 se resume el desempeño promedio de los participantes en las medidas cognitivas de FE, encontrándose en la mayoría de las tareas un rendimiento dentro de lo esperado para la edad. 
Tabla 1. Resumen de las puntuaciones de los participantes en las tareas de FE

\begin{tabular}{lll}
\hline Dominios de FE/Tareas & $M$ & DT \\
\hline Control inhibitorio & & \\
$\quad$ Stroop - Interferencia & 51.6 & 11.3 \\
Memoria de trabajo & & \\
$\quad$ Aritmética & 6.0 & 3.1 \\
$\quad$ Dígitos & 8.7 & 2.8 \\
$\quad$ Letras y números & 8.1 & 3.2 \\
$\quad$ IMT (WAIS-III) & 82.46 & 15,4 \\
Flexibilidad cognitiva & & \\
$\quad$ Número de errores perseverativos en el M-WCST & 45.5 & 12.7 \\
Categorización & & \\
$\quad$ Número de categorías en el M-WCST & 45.6 & 12.5 \\
\hline
\end{tabular}

M: Media; DT: Desviación Típica; IMT: índice de memoria de trabajo; M-WCST: Test de Clasificación de Tarjetas de Wisconsin Modificado.

El análisis de correlación de Pearson entre el rendimiento académico y las distintas medidas cognitivas de FE evidencia que existe una relación positiva estadísticamente significativa entre el rendimiento académico de estudiantes universitarios y su desempeño en la tarea de memoria de trabajo verbal Aritmética $(\mathrm{r}=.350, p=.000)$, así como también, entre rendimiento académico y el número de errores perseverativos en el M-WCST ( $\mathrm{r}=.207, p=.018)$, una medida de flexibilidad cognitiva.

El modelo de regresión jerárquica múltiple mostró que la tarea de memoria de trabajo verbal Aritmética, la medida de flexibilidad cognitiva (número de errores perseverativos en el M-WCST) y la medida de categorización (número de categorías de M-WCST), son los mejores predictores del rendimiento académico de estudiantes universitarios en la muestra estudiada $F(3)$ $=8.239, p=.000, \eta_{\mathrm{p}}^{2}=.198$ (ver Tabla 2). Ahora bien, el tamaño del efecto para este modelo es pequeño, logrando explicar el $19,8 \%$ de la varianza. La ecuación predictora del modelo sería: Rendimiento académico $=3.107+(0.011 *$ Número de errores perseverativos en el M-WCST $)+$ $(0.040 *$ Aritmética $)+(-0.008 *$ Número de categorías en el M-WCST) . 
Tabla 2. Coeficientes tipificados y probabilidad del modelo

\begin{tabular}{lcccccc} 
& $\begin{array}{c}\text { R } \\
\text { cuadrado }\end{array}$ & B & SE B & $\begin{array}{c}\text { B } \\
\text { estandarizado }\end{array}$ & t & $p$ \\
\hline Constante & .198 & 3.107 & .139 & & 22.298 & .000 \\
Aritmética & & .040 & .010 & .348 & 3.886 & .000 \\
No. de errores perseverativos en el & & .011 & .004 & .393 & 3.073 & .003 \\
M-WCST & & & & & & \\
No. de categorías en el M-WCST & & -.008 & .004 & -.279 & -2.179 & .032 \\
\hline
\end{tabular}

Adicionalmente, se realizaron comparaciones entre varones y muejeres, y entre grupos con rendimiento académico promedio y bajo rendimiento académico en las tareas cognitivas de FE con el fin de identificar diferencias significativas relacionadas con el desempeño académico global en memoria de trabajo, control inhibitorio, flexibilidad cognitiva y categorización (ver Tabla 3 y 4$)$.

Tabla 3. Diferencias en FE entre varones y mujeres

\begin{tabular}{|c|c|c|c|c|c|c|}
\hline Dominio FE/Tarea & Grupo & $M e$ & DT & $U$ de Mann-Whitney & Sig. & $\mathbf{r}$ \\
\hline \multicolumn{7}{|l|}{ Control inhibitorio } \\
\hline \multirow{2}{*}{ Stroop - Interferencia } & $\mathrm{V}$ & 52.0 & 11.3 & 955.00 & .164 & 0.41 \\
\hline & M & 50.0 & 11.3 & & & \\
\hline \multicolumn{7}{|l|}{ Memoria de trabajo } \\
\hline \multirow{2}{*}{ Aritmética } & V & 6.0 & 2.9 & 863.000 & .040 & 0.37 \\
\hline & M & 4.5 & 2.9 & & & \\
\hline \multirow{2}{*}{ Dígitos } & $\mathrm{V}$ & 9.0 & 2.8 & 1138.500 & .924 & 0.49 \\
\hline & M & 9.0 & 2.8 & & & \\
\hline \multirow{2}{*}{ Letras y números } & $\mathrm{V}$ & 9.0 & 3.6 & 898.000 & .071 & 0.39 \\
\hline & M & 7.0 & 3.0 & & & \\
\hline \multirow{2}{*}{ IMT (WAIS-III) } & $\mathrm{V}$ & 81.0 & 16.5 & 914.500 & .094 & 0.39 \\
\hline & M & 78.0 & 13.7 & & & \\
\hline \multicolumn{7}{|l|}{ Flexibilidad cognitiva } \\
\hline \multirow{2}{*}{$\begin{array}{l}\text { Errores perseverativos } \\
\text { WCST-M }\end{array}$} & $\mathrm{V}$ & 45.0 & 12.5 & 1018.000 & .343 & 0.44 \\
\hline & M & 44.0 & 12.7 & & & \\
\hline \multicolumn{7}{|l|}{ Categorización } \\
\hline \multirow{2}{*}{$\begin{array}{l}\text { Número de categorías } \\
\text { WCST-M }\end{array}$} & $\mathrm{V}$ & 53.0 & 12.2 & 1142.000 & .943 & 0.49 \\
\hline & M & 53.0 & 13.0 & & & \\
\hline
\end{tabular}

Me: Mediana; DT: Desviación Típica; r: Tamaño del efecto; V: varon; M: Mujer 
Al comparar a los participantes en función del sexo, se encontró diferencia estadísticamente significativas entre varones y mujeres en la tarea de memoria de trabajo verbal Aritmética $(\mathrm{U}=863.000, p=.040 . \mathrm{r}=.37)$, con un tamaño del efecto mediano. De igual forma, en la comparación de los grupos de estudiantes con rendimiento académico promedio y bajo rendimiento académico se encontró diferencia estadísticamente significativa en esta tarea (U=826.500 $p=.001$. $\mathrm{r}=.32$ ), con un tamaño del efecto mediano.

Tabla 4. Diferencias en FE entre estudiantes con rendimiento académico bajo y promedio.

\begin{tabular}{|c|c|c|c|c|c|c|}
\hline Dominio FE/Tarea & Grupo & $M e$ & DT & $U$ de Mann-Whitney & Sig. & $\mathbf{r}$ \\
\hline \multicolumn{7}{|l|}{ Control inhibitorio } \\
\hline \multirow{2}{*}{ Stroop - Interferencia } & RAP & 52.0 & 11.3 & 1153.500 & .221 & 0.11 \\
\hline & RAB & 50.0 & 11.3 & & & \\
\hline \multicolumn{7}{|l|}{ Memoria de trabajo } \\
\hline \multirow{2}{*}{ Aritmética } & RAP & 6.0 & 2.9 & 826.500 & .001 & 0.32 \\
\hline & RAB & 5.0 & 2.9 & & & \\
\hline \multirow{2}{*}{ Dígitos } & RAP & 9.0 & 2.8 & 1259.000 & .650 & 0.04 \\
\hline & $\mathrm{RAB}$ & 9.0 & 2.8 & & & \\
\hline \multirow{2}{*}{ Letras y números } & RAP & 9.0 & 3.6 & 1166.500 & .287 & 0.10 \\
\hline & $\mathrm{RAB}$ & 7.0 & 3.0 & & & \\
\hline \multirow{2}{*}{ IMT (WAIS-III) } & RAP & 81.0 & 16.5 & 1089.000 & .117 & 0.15 \\
\hline & $\mathrm{RAB}$ & 77.0 & 13.7 & & & \\
\hline \multicolumn{7}{|l|}{ Flexibilidad cognitiva } \\
\hline \multirow{2}{*}{$\begin{array}{l}\text { Errores perseverativos } \\
\text { WCST-M }\end{array}$} & RAP & 51.0 & 12.5 & 1142.000 & .221 & 0.12 \\
\hline & $\mathrm{RAB}$ & 45.0 & 12.7 & & & \\
\hline \multicolumn{7}{|l|}{ Categorización } \\
\hline \multirow{2}{*}{$\begin{array}{l}\text { Número de categorías } \\
\text { WCST-M }\end{array}$} & RAP & 53.0 & 12.2 & 1259.500 & .650 & 0.04 \\
\hline & RAB & 53.0 & 13.0 & & & \\
\hline
\end{tabular}

Me: Mediana; DT: Desviación Típica; r: Tamaño del efecto; RAP: rendimiento académico promedio; RAB: rendimiento académico bajo; IMT: índice de memoria de trabajo.

\section{Discusión}

Estudiamos la relación entre FE y rendimiento académico, analizando mediante medidas cognitivas de FE si la memoria de trabajo, el control inhibitorio, la flexibilidad cognitiva, y la capacidad de abstracción y formación de conceptos predicen el logro académico general de estudiantes universitarios. El modelo de regresión jerárquica múltiple permitió identificar tres $\mathrm{FE}$ cognitivas que predicen el rendimiento académico de estudiantes universitarios: memoria de trabajo verbal, flexibilidad cognitiva y categorización; si bien, su asociación es de debil a mediana 
y por tanto su capacidad para predecir el rendimiento académico es limitada. Estos hallazgos son similares a lo descrito en el trabajo de Dubuc et al. (2020) en el que se estudió la relación entre dos procesos ejecutivos, memoria de trabajo y control inhibitorio, y el rendimiento académico en adolescentes, encontrandose que existe una debil asociación entre estas variables y por tanto tienen una pobre capacidad para predecir variaciones en el desempeño escolar.

Ahora bien, de las tres variables cognitivas que predicen el rendimiento académico en este estudio, solo la memoria de trabajo verbal tuvo una asociación mediana. Esto es coherente con el hecho de que en estudios previos la memoria de trabajo ha sido fuertemente implicada en el rendimiento académico, y su papel en los procesos de aprendizaje ha sido ampliamente documentado en la infancia (Demetriou, Kazali, Kazi, \& Spanoudis, 2020; Ramos et al., 2018; Reyes et al., 2015; Simone, et al., 2018; Swanson, \& Alloway, 2012; Visu-Petra et al., 2011). El presente trabajo es una contribución al estudio de esta asociación en población universitaria.

Al comparar el desempeño de estudiantes con rendimiento académico promedio y bajo rendimiento académico, se encontró diferencias estadísticamente significativas entre los grupos en la tarea de memoria de trabajo verbal Aritmética, con un tamaño del efecto moderado. Así, nuestros resultados indican que la capacidad para retener temporalmente información en la memoria y manipularla mentalmente para solucionar problemas está asociada al logro académico general en población universitaria (Ramos et al., 2018; Reyes et al., 2015; Simone, et al., 2018; Swanson, \& Alloway, 2012; Van der Ven et al., 2012; Visu-Petra et al., 2011; Wiest, Wong, Bacon, Rosales, \& Wiest, 2020). La tarea Aritmética tiene una demanda importante de selección y funcionamiento de una estrategia, así como de mantenimiento y alternancia de la atención para resolver un problema; este es el componente ejecutivo de la memoria de trabajo. La memoria de trabajo facilita el seguimiento de instrucciones, el sostenimiento y selectividad de la atención, el control de la calidad del propio trabajo y la generación de nuevas soluciones a los problemas, capacidades indispensables para el éxito en los procesos de aprendizaje (Gathercole, \& Alloway, 2008; Prosen \& Smrtnik Vitulić, 2014).

De acuerdo a lo descrito en investigaciones previas como el trabajo de Lynn e Irwing (2008), los varones tienen un mejor desempeño a nivel de memoria de trabajo que las muejeres. En ese estudio se consideró la subprueba Aritmética de la escala de inteligencia para adultos de Wechsler WAIS-III como medida de memoria de trabajo verbal. En nuestro estudio desarrollado en la Universidad Tecnológica de Bolívar también se encontró diferencia significativa entre varones y mujeres en la memoria de trabajo verbal evaluada mediante la tarea Aritmética. Estos hallazgos discrepan con lo descrito por Alarcón, Cservenka, Fair, y Nagel (2014), Dubuc et al. (2020), Hsu et al. (2015), e Ishak et al. (2012), quienes no han encontraron diferencias significativas entre varones y mujeres en la capacidad de memoria de trabajo. Es probable que las discrepancias en los resultados de estas investigaciones se expliquen en parte por la variedad de tareas utilizadas para medir la capacidad de memoria de trabajo en los diferentes estudios (Grissom \& Reyes, 2019; Voyer, Voyer, \& Saint-Aubin, 2017).

La presente investigación también mostró que la flexibilidad cognitiva y la capacidad de abstracción y formación de conceptos están relacionadas con el rendimiento académico, si bien esta asociación es débil (Espy et al., 2004). Esto implica que aquellos estudiantes que tienen una mayor habilidad para cambiar entre un set de respuestas diferentes, ya sea de pensamientos o de acciones, en respuesta a las demandas de una situación; y que tienen una mayor habilidad para abstraer conceptos, podrían tener un mayor logro académico en el contexto universitario. Estas 
capacidades les permiten a los estudiantes "aprender de los errores, generar estrategias alternativas, dividir la atención y procesar la información actual” (Anderson, 2002 citado por Ramírez, \& Ostrosky, 2012, p. 583). La flexibilidad cognitiva, y la capacidad de abstracción y formación de conceptos, no se han estudiado mucho en el contexto universitario en relación con el rendimiento académico, pero nuestro resultado está en línea con investigaciones anteriores que han demostrado que son predictores de éxito escolar (McKee, 2017); en contraposición con lo descrito por Barceló et al. (2006) y Van der Ven et al. (2012).

En este trabajo no se encontró relación entre control inhibitorio y rendimiento académico, similar a lo descrito por Dubuc et al. (2020) y en contraposición a los hallazgos de estudios con población infantil (Visu-Petra et al., 2011; Samuels et al., 2016). Teniendo en cuenta que la edad puede estar mediando la influencia de las FE en el rendimiento académico debido a que el córtex prefrontal y sus conexiones alcanzan mayor desarrollo durante la adolescencia y adultez temprana (Blakemore \& Choudhury, 2006; Gogtay et al., 2004; Oyefiade et al., 2018), futuras investigaciones podrían estudiar el papel de la edad en relación con la influencia que tienen FE como el control inhibitorio en el rendimiento académico de estudiantes universitarios.

Si bien existen estudios previos en población universitaria en los que se ha encontrado que las FE son predictores del rendimiento académico (Knouse et al., 2014; Baars et al., 2015), en esos trabajos se han utilizado principalmente medidas de autorreporte sobre FE que exploran, en el marco de actividades de la vida diaria, procesos de planeación, atención, autocontrol/automonitoreo, autoadministración del tiempo, auto-organización y solución de problemas, automotivación, y autorregulación emocional; es decir, son procesos de ejecución conductual que aunque relacionados, son diferentes de la ejecución cognitiva que usualmente es estudiada mediante tareas de laboratorio como las utilizadas en nuestro estudio (Barkley, 2012). De allí que nuestro trabajo haga un importante aporte a la identificación de predictores del rendimiento académico desde medidas cognitivas de FE.

En conclusión, este estudio muestra que FE cognitivas como memoria de trabajo verbal, flexibilidad cognitiva y categorización predicen el rendimiento académico general de estudiantes universitarios, si bien el modelo explica solo el 19\% de la varianza. Al comparar el desempeño de estudiantes con rendimiento académico promedio y bajo rendimiento académico, se encontró que la memoria de trabajo verbal está asociada al logro académico general en población universitaria, y que los hombres tienen un mejor rendimiento que las mujeres en esta habilidad cognitiva, siendo esta diferencia mediana. Estos resultados refuerzan la idea de la pertinencia de intervenir en el desarrollo de las FE, en particular de la memoria de trabajo, desde la edad escolar, de manera que los estudiantes desarrollen capacidades que favorezcan su desempeño académico a lo largo de la vida (Wiest et al., 2020); y que este entrenamiento ayude a cerrar la brecha que puede existir entre varones y mujeres en el rendimiento académico general (Voyer \& Voyer, 2014).

Nuestros hallazgos ponen de relieve la necesidad de explorar otras variables cognitivas, conductuales y psicosociales que pudieran estar influyendo también en el rendimiento académico general (Asikainen, 2018; Asikainen, Hailikari, \& Mattsson, 2018) e incluirlas en un modelo multivariable para el diseño de programas de intervención desde las dependencias de bienestar universitario que sean más efectivos para promover la permanencia estudiantil. Futuros estudios deberán considerar la edad como posible variable mediadora de la relación entre FE y rendimiento académico general, de igual manera, se pueden incluir factores psicosociales asociados 
al rendimiento académico en modelos explicativos más complejos del desempeño académico en el contexto universitario.

En cuanto a las limitaciones de nuestro estudio, señalar la conveniencia de ampliar el tamaño muestral e incluir adicionalmente medidas de FE conductuales que resulten ser más ecológicas que las tareas cognitivas de laboratorio. Otra limitación es el número de medidas cognitivas usadas. No se realiza una evaluación neuropsicológica integral que incluya múltiples medidas verbales y no verbales de FE, por tanto los resultados deben interpretarse con precaución. Adicionalmente, en este trabajo no se establecen diferencias entre facultades o programas académicos a los que pertenecen los participantes.

\section{Referencias}

Alarcón, G., Cservenka, A., Fair, D. A., \& Nagel, B. J. (2014). Sex differences in the neural substrates of spatial working memory during adolescence are not mediated by endogenous testosterone. Brain research, 1593, 40-54.

Asikainen, H. (2018). Examining indicators for effective studying-The interplay between student integration, psychological flexibility and self-regulation in learning. Psychology, Society, \& Education, 10(2), 225-237.

Asikainen, H., Hailikari, T., \& Mattsson, M. (2018). The interplay between academic emotions, psychological flexibility and self-regulation as predictors of academic achievement. Journal of Further and Higher Education, 42(4), 439-453.

Baars, M. A., Nije Bijvank, M., Tonnaer, G. H., \& Jolles, J. (2015). Self-report measures of executive functioning are a determinant of academic performance in first-year students at a university of applied sciences. Frontiers in psychology, 6, 1131.

Baddeley, A. (2012). Working memory: theories, models, and controversies. Annual review of psychology, 63, 1-29.

Barceló, E., Lewis. S. \& Moreno, M. (2006). Funciones ejecutivas en estudiantes universitarios que presentan bajo y alto rendimiento académico. Psicología desde el Caribe, (18), 109-138.

Barkley, R. A. (2012). The Barkley deficits in executive functioning scale. New York: Guilford Press.

Barreto, L. C. R., Roa, C. A. P., \& del Carmen Pulido, N. (2016). Propiedades psicométricas del Stroop, test de colores y palabras en población colombiana no patológica. Universitas Psychologica, 15(2), 255-272.

Blakemore, S. J., \& Choudhury, S. (2006). Development of the adolescent brain: implications for executive function and social cognition. Journal of child psychology and psychiatry, 47(3-4), 296-312.

Casey, B. J., Getz, S., \& Galvan, A. (2008). The adolescent brain. Developmental. Review. 28, 62-77. doi: 10.1016/j.dr.2007.08.003

Cerda, G., Pérez, C., Romera, E.M., Casas, J.A., \& Ortega-Ruiz, R. (2017). Influence of cognitive and motivational variables in academic mathematics performance in chilean students. Educacion XX1 20(2), pp. 365-385

Demetriou, A., Kazali, E., Kazi, S., \& Spanoudis, G. (2020). Cognition and cognizance in preschool predict school achievement in primary school. Cognitive Development, 54, 100872. 
Dubuc, M. M., Aubertin-Leheudre, M., \& Karelis, A. D. (2020). Relationship between interference control and working memory with academic performance in high school students: The Adolescent Student Academic Performance longitudinal study (ASAP). Journal of Adolescence, 80, 204-213.

Espy, K., McDiarmid, M., Cwik, M., Stalets, M., Hamby, A. \& Senn, T. (2004). The contribution of executive functions to emergent mathematics skills in preschool children. Developmental Neuropsychology, 26, 465-486

García-Molina, A., Enseñat, A., Tirapu-Ustárroz, J., \& Roig-Rovira J. (2009). Maduración de la corteza prefrontal y desarrollo de las funciones ejecutivas durante los primeros cinco años de vida. Revista de Neurología, 48, 435-440.

Gathercole, S., \& Alloway, T. P. (2008). Working memory and learning: A practical guide for teachers. Sage.

Giedd, J. N., \& Rapoport, J. L. (2010). Structural MRI of pediatric brain development: what have we learned and where are we going? Neuron 67, 728-734. doi: 10.1016/j.neuron.2010.08.040

Gogtay, N., Giedd, J.N., Lusk, L., Hayashi, K.M., Greenstein, D., ... \& Thompson, P.M. (2004). Dynamic mapping of human cortical development during childhood through early adulthood. Proceedings of the National Academy of Sciences of the United States of America, 101, 8174-8179.

Golden, C. J. (1999). Stroop: Test de colores y palabras. Madrid: Tea.

Gómez-Pérez, E., Ostrosky-Solís, F., \& Próspero-García, O. (2003). The development of attention, memory and the inhibitory processes: The chronological relation with the maturation of brain structure and functioning. Revista de Neurología 37(6), 561-567

Grissom, N. M., \& Reyes, T. M. (2019). Let's call the whole thing off: evaluating gender and sex differences in executive function. Neuropsychopharmacology, 44(1), 86-96.

Heaton, R.K., Chelune, G.J., Talley, J.L., Kay, G.G., \& Gurtiss, G. (1993). Wisconsin Card Sorting Test Manual. Odessa. FL: Psychological Assessment Resources.

Hsu, H. L., Chen, D. Y. T., Tseng, Y. C., Kuo, Y. S., Huang, Y. L., Chiu, W. T., ... \& Chen, C. J. (2015). Sex differences in working memory after mild traumatic brain injury: a functional MR imaging study. Radiology, 276(3), 828-835.

Iniesta, A.V., López-López, J.A., Corbí, R.G., Pérez, P.M., \& Costa, J.L.C. (2017). Differences in cognitive, motivational and contextual variables between under-achieving, normallyachieving, and over-achieving students: A mixed-effects analysis. Psicothema 29(4), 533-538

Ishak, I., Jufri, N. F., Lubis, S. H., Saat, N. Z. M., Omar, B., Arlin, R., ... \& Mohamed, N. (2012). The study of working memory and academic performance of Faculty of Health Sciences students. Procedia-Social and Behavioral Sciences, 60, 596-601.

Knouse, L., Feldman, G. \& Blevins, E. (2014). Executive functioning difficulties as predictors of academic performance: Examining the role of grade goals. Learning and Individual Differences, 36, 19-26.

Lynn, R., \& Irwing, P. (2008). Sex differences in mental arithmetic, digit span, and g defined as working memory capacity. Intelligence, 36(3), 226-235.

McKee, J. (2017). Executive Functions and Resilience in First-Year Undergraduate Students (Doctoral dissertation, University of Calgary). 
Miyake, A., Friedman, N. P., Emerson, M. J., Witzki, A. H., Howerter, A., \& Wager, T. D. (2000). The unity and diversity of executive functions and their contributions to complex "frontal lobe" tasks: A latent variable analysis. Cognitive Psychology, 41, 49-100.

Oyefiade, A. A., Ameis, S., Lerch, J. P., Rockel, C., Szulc, K. U., Scantlebury, N., ... \& Mabbott, D. J. (2018). Development of short-range white matter in healthy children and adolescents. Human brain mapping, 39(1), 204-217.

Prosen, S. \& Smrtnik Vitulić, H. (2014). Executive function in different groups of university students. Review of psychology, 21(2), 0-143.

Ramírez, F. M. J., \& Ostrosky, F. (2012). Flexibilidad cognitiva después de un traumatismo craneoencefálico. Acta de investigación psicológica, 2(1), 582-591.

Ramos,C.,Jadán-Guerrero,J.,\& Gómez-García,A.(2018). Relaciónentreelrendimientoacadémico y el autorreporte del funcionamiento ejecutivo de adolescentes ecuatorianos. Avances en Psicología Latinoamericana, 36(2), 405-417.

Reyes, S., Barreyro, J. \& Injoque-Ricle, I. (2015). El rol de la función ejecutiva en el rendimiento académico en niños de 9 años. Revista Neuropsicología Latinoamericana, 7(2), 42-72

Rose, D. \& Rose, K. (2007). Deficits in Executive Function Processes: A Curriculum-based Intervention. In Lynn Meltzer (Ed.). Executive Function in Education: From Theory to Practice. New York: Guilford Publications.

Samuels, W., Tournaki, N., Blackman, S. \& Zilinski, C. (2016). Executive functioning predicts academic achievement in middle school: A four-year longitudinal study. The Journal of Educational Research, 109(5), 478-490.

Santa-Cruz, C., \& Rosas, R. (2017). Mapping of Executive Functions/Cartografía de las Funciones Ejecutivas. Estudios de Psicología, 38(2), 284-310.

Simone, A. N., Marks, D. J., Bédard, A. C., \& Halperin, J. M. (2018). Low working memory rather than ADHD symptoms predicts poor academic achievement in school-aged children. Journal of abnormal child psychology, 46(2), 277-290.

Swanson, H. L., \& Alloway, T. P. (2012). Working memory, learning, and academic achievement APA educational psychology handbook, Vol 1: Theories, constructs, and critical issues. Washington, DC, US: American Psychological Association.

Van der Ven, S. H., Kroesbergen, E. H., Boom, J., \& Leseman, P. P. (2012). The development of executive functions and early mathematics: A dynamic relationship. British Journal of Educational Psychology, 82(1), 100-119.

Visu-Petra, L., Cheie, L., Benga, O., \& Miclea, M. (2011). Cognitive control goes to school: The impact of executive functions on academic performance. Procedia-Social and Behavioral Sciences, 11, 240-244.

Voyer, D., \& Voyer, S. D. (2014). Gender differences in scholastic achievement: A meta-analysis. Psychological Bulletin, 140(4), 1174-1204. https://doi.org/10.1037/a0036620

Voyer, D., Voyer, S. D., \& Saint-Aubin, J. (2017). Sex differences in visual-spatial working memory: A meta-analysis. Psychonomic bulletin \& review, 24(2), 307-334.

Wechsler, D. (2001). WAIS-III. Escala de Inteligencia de Wechsler para Adultos. Madrid: TEA Ediciones.

Wiest, D. J., Wong, E. H., Bacon, J. M., Rosales, K. P., \& Wiest, G. M. (2020). The Effectiveness of Computerized Cognitive Training on Working Memory in a School Setting. Applied Cognitive Psychology, 34(2), 465-471. 